\title{
Mediating Effects of Smartphone Utilization between Attitude and Willingness to Use Home-Based Healthcare ICT among Older Adults
}

\author{
Heui Sug Jo, Yu Seong Hwang, Yuliya Dronina \\ Department of Health Policy and Management, Kangwon National University School of Medicine, Chuncheon, Korea
}

Objectives: This study explored the direct and indirect effects of knowledge of new technology (e.g., artificial intelligence, the Internet of Things, and the Fourth Industrial Revolution), attitudes towards technology use, and smartphone utilization skills on older adults' willingness to use home-based information and communication technology (ICT) for self-health management. Methods: A phone survey was conducted among 300 older adults aged 65 or older in Gangwon Province, Republic of Korea. A path analysis was performed to identify the direct and indirect effects of knowledge of new technology, attitudes towards technology use, and smartphone utilization skills on willingness to use home-based healthcare ICT. Socioeconomic variables were used as control variables. Results: Knowledge of new technology, but not attitudes towards technology use, had a direct impact on smartphone utilization skills. Attitude towards technology use and smartphone utilization skills showed significant effects on willingness to use home-based healthcare ICT. One standard unit change in attitudes towards technology use contributed to a 0.172 unit change in willingness $(p=0.001)$, and one standard unit change in smartphone utilization skills changed willingness by 0.246 units $(p<0.001)$. In addition, older adults with a higher education level and economic status, and lower self-related health status, were more willing to use home-based healthcare ICT. Conclusions: These findings underscore the necessity of enhancing the smartphone utilization skills of older adults and attitudes towards technology use. Providing more user-friendly services and increasing smartphone utilization skills among older adults would contribute to willingness to use home-based ICT for healthcare management.

Keywords: Aging, Home Care Services, Information Technology, Internet of Things, Artificial Intelligence

Submitted: June 12, 2020

Revised: 1st, December 24, 2020; 2nd, February 17, 2021; 3rd, April 3, 2021 Accepted: April 3, 2021

\section{Corresponding Author}

Yuliya Dronina

Department of Health Policy and Management, Kangwon National University School of Medicine, Chuncheon 24341, Korea. Tel: +8233-250-8910,E-mail: julia_dronina@yahoo.com (https://orcid.org/ 0000-0002-5912-3553)

This is an Open Access article distributed under the terms of the Creative Commons Attribution Non-Commercial License (http://creativecommons.org/licenses/by$\mathrm{nc} / 4.0 /$ ) which permits unrestricted non-commercial use, distribution, and reproduction in any medium, provided the original work is properly cited.

(C) 2021 The Korean Society of Medical Informatics

\section{Introduction}

The rapid increase of the population of adults aged 65 years or older in South Korea is causing issues related to population aging, which in turn necessitates the provision of appropriate and sustainable health services to older adults [1]. However, the emergence of coronavirus diseases 2019 (COVID-19) has led to the need to provide medical services remotely [2]. Home-based healthcare is a resourceful way of providing health services distantly, ensuring the safety of older adults. Furthermore, older adults generally prefer to receive appropriate healthcare services at home, rather than staying at a hospital or long-term facility, resulting in the 
need to develop "aging in place" services [3]. In this sense, the development and ubiquity of information and communication technology (ICT) could help solve issues connected to the aging population.

The widespread use of ICT has contributed to its introduction to healthcare, shifting from a doctor-centered to a patient-centered approach. Several solutions have recently been implemented to provide ICT-based health service improvements for older adults, such as telemedicine applications, wearable devices to register health information, and robotic assistive systems [4]. Previous studies have reported positive effects of ICT-based health technology in the monitoring and support of older adults [5,6]. In particular, older adults reported satisfaction with using a telemedicine system to improve chronic disease management; however, it did not decrease the number of hospitalizations [4]. Recently, home-based ICT has been incorporated in a wide range of applications in the development of assistive technology (AT) services for older adults [7].

Previous studies have discussed the implementation and potential benefits and challenges of smart home AT services, robotics systems, and wearable monitoring devices [8]. Intervention studies have reported positive effects and impressions on the users of the proposed solutions [9]. Smart home AT services can provide useful and comprehensive information on older people's health status that can help in tracking their diseases and health conditions [10]. Furthermore, rapid adoption of this new technology by older adults will contribute to their integration into today's information society, where information plays a crucial role. However, a previous study reported difficulties in the adoption of new technology by this population and highlighted the need to educate them about technology [11]. This issue is directly related to digital and eHealth literacy, and older adults' propensity to use new technologies in this domain [12].

Digital literacy and the understanding of new ICT directly influence the utilization of technologies by older adults. A study found that although most digital technologies are designed for younger generations, the experience of using technology helped to decrease technology use anxiety among older adults [13]. Another study reported that older people considered buying digital technologies (e.g., computers) that were more appropriate for young people than for them [14]. The purpose of this study was to examine how older adults' knowledge of new technology, such as artificial intelligence (AI), the Internet of Things (IoT), and the Fourth Industrial Revolution (4IR), and attitudes towards the use of technology influenced their smartphone utilization skills and will- ingness to use home-based healthcare ICT products. That is, this research had two objectives: (1) to investigate the direct effect of older adults' knowledge of new technology and attitude towards the use of technology on their willingness to use home-based healthcare ICT and smartphone utilization skills, and (2) to analyze the indirect effect of older adults' knowledge of new technology and attitude towards using technology on their willingness to use home-based healthcare ICT and the relationship between their smartphone utilization skills and willingness to use home-based healthcare ICT.

\section{Methods}

\section{Study Design}

The study used multivariable path analysis to simultaneously estimate the relationships between the predictor variables (attitude towards the use of technology and knowledge of new technology), the mediating variable (smartphone utilization skills), and willingness to use home-based healthcare ICT by older adults.

In light of the study purpose, the information-motivationbehavior skills (IMB) model was adapted to evaluate the willingness to use home-based healthcare ICT services among older adults (Figure 1). The IMB model developed by Fisher and Fisher [15] was used to evaluate the impact of health behavior and conditions on health behavior changes. The study framework was modified as follows: (1) knowledge of new technology was considered to correspond to the information component of the IMB model; (2) attitude towards the use of technology was employed to evaluate motivation; (3) smartphone utilization skills were a measurement of behavioral skills; and (4) willingness to use home-based healthcare ICT

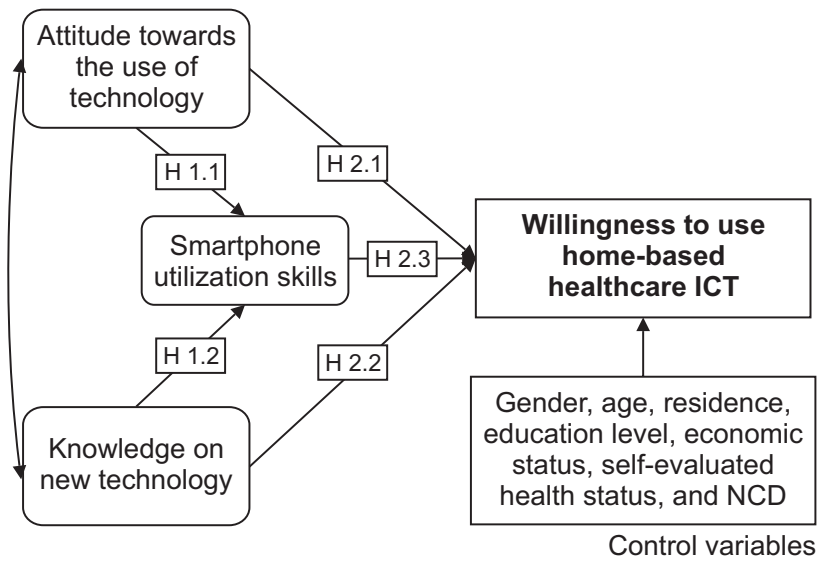

Figure 1. Study framework. ICT: information and communication technology, NCD: non-communicable disease. 
was a measurement of behavior change. Willingness to use home-based healthcare ICT was also explored based on socioeconomic factors and health status [16]. We hypothesized that [11]:

1) A positive attitude towards the use of technology would positively influence older adults' smartphone utilization skills (H1.1);

2) Knowledge of new technology would positively influence older adults' smartphone utilization skills (H1.2);

3) A positive attitude towards the use of technology would positively influence older adults' willingness to use home-based healthcare ICT (H2.1);

4) Knowledge of new technology would positively influence older adults' willingness to use home-based healthcare ICT (H2.2);

5) Smartphone utilization skills would positively influence older adults' willingness to use home-based healthcare ICT (H2.3).

\section{Measurements}

For data collection, a questionnaire including questions on ICT literacy adopted from the 2018 Digital Divide Survey was developed. Willingness to use home-based healthcare ICT was evaluated by asking the question, "What do you think about using information and communication technology to take care of your health when you are at home?" This question was answered based on a 5-point Likert scale $(1=$ totally useless, 5 = very useful).

Knowledge of new technology and attitudes towards the use of technology were selected as the predicting variables, and smartphone utilization skills were used as the mediating variable. Knowledge of new technology was assessed through the question, "How would you evaluate your knowledge about the Fourth Industrial Revolution, artificial intelligence and the Internet of Things?", also on a 5-point Likert scale. Attitudes towards technology use were assessed by asking participants five questions about the usefulness of technology, their perceptions of using and joining the information society, and the potential hazardous effects and difficulties of joining the information society. A 5-point Likert scale was applied to assess opinions about potential hazardous effects. The item dealing with "difficulties of integrating into the information society" was reverse-coded. Smartphone utilization skills were evaluated using 11 questions. Participants were asked about using a smartphone and about some of the functions and services of smartphones. Data were coded on a 10-point scale, where non-users were coded as 0 and users of all services and applications provided in the questionnaire were coded as 10 .

Socioeconomic data included sex, age, residence, education level, and economic status. Sex was coded as male (0) or female (1), and residence was divided into urban (0) and rural (1). Education level was coded into four groups: uneducated (0), primary school (1), secondary school (2), and high school or higher (3). Economic status was assessed using a 5 -point Likert scale ( $1=$ very poor, $5=$ wealthy $)$ and health status was assessed using a 5-point Likert scale self-report ( $1=$ very poor, $5=$ healthy). Non-communicable diseases (NCDs) were coded as a discrete variable where 0 indicated the absence of NCDs.

\section{Participants}

The sample size was calculated based on the assumption that an adequate sample size should be 10 times the number of parameters in the path analysis, and the best sample size should be 20 times the number of parameters in the path analysis. Wolf et al. [17] suggested a range of sample size requirements, ranging from 30 to 460 cases depending on statistical power, the bias in the parameter estimates, and overall solution propriety. A total of 300 older adults participated in the survey, which was conducted by a specialized research company named Korea Data Network (KDN). The survey panel data of KDN were used to select the potential participants. Potential participants were screened by telephone for eligibility and willingness to participate and selected by the following criteria: (1) aged 65 or older; (2) having made at least one visit to a hospital in the last 3 years; (3) residence in Gangwon Province.

The survey questionnaire and guidelines were developed and updated for easy understanding by older adults and provided to KDN. A phone panel survey was conducted from October 28, 2019 to November 22, 2019 and informed consent was obtained from the study participants by verbal agreement to participate in the survey. The study's protocol was approved by the Institutional Review Board of Kangwon National University (No. 2019-05-006-005).

\section{Data Analysis}

The sociodemographic characteristics of the study's participants were analyzed. A path analysis was performed to identify the relationship between willingness to use home-based healthcare ICT and knowledge of new technology (information), attitudes towards the use of technology (motivation), and smartphone utilization skills (behavior skills). Direct relationships were calculated as the path regression coefficient between two variables. Indirect relationships were calculated 
as the product of the regression coefficients for the relationships or linkages in the path.

We did not pursue a more conventional model building or model comparison approach, often associated with structural equation modeling, for the following reasons. First, since our analysis focused on a count-dependent variable, traditional goodness-of-fit statistics such as the root mean square error of approximation, comparative fit index (CFI), the Tucker-Lewis index (TLI) were not appropriate to assess the fit of our model. Second, the main objective was to evaluate whether knowledge of new technology and attitudes towards the use of technology variables were significantly, directly, and indirectly associated with willingness to use home-based healthcare ICT, rather than examining the fit of alternative models. However, the CFI and TLI results suggested a reasonably good fit for the measurement portion of the model.

The sociodemographic characteristics and health status of the participants were included in these analyses as control variables. Data analysis was performed using SPSS version 24.0 and AMOS version 18.0 (IBM Corp., Armonk, NY,
USA).

\section{Results}

\section{Descriptive Statistics of the Study Population}

Table 1 shows the descriptive statistics of the study population. Participants' mean age was 74.2 years, with a standard deviation of 5.45 years. The proportion of women was higher than that of men (55.7\% vs. $44.3 \%)$ and there were more participants from urban areas than rural areas $(53 \%$ and $46 \%$, respectively). Furthermore, $15.7 \%$ of the participants were uneducated, $24.7 \%$ had finished primary school, $27.3 \%$ had completed secondary school education, and $32.3 \%$ had a high school or higher education. Most participants evaluated their economic status as average (mean \pm standard deviation, $3.2 \pm 0.75$ ); their self-evaluated health status was also scored as average (3.18 \pm 0.95$)$. The average number of NCDs was $2 \pm 1.32$. Knowledge of new technology was low $(2.26 \pm 1.06$, using a 5-point scale). Attitudes towards the use of technology were average, with a score of 3.22 out of 5 . Smartphone

Table 1. Descriptive statistics of the study population

\begin{tabular}{lcccc}
\hline \multicolumn{1}{c}{ Variable } & Frequency & Mean \pm SD (\%) & Kurtosis & Skewness \\
\hline Willingness to use home-based healthcare ICT & 300 & $3.49 \pm 0.82$ & -0.516 & -0.180 \\
Knowledge of new technology & 300 & $2.26 \pm 1.06$ & -0.605 & 0.542 \\
Attitudes towards the use of technology & 300 & $3.22 \pm 0.47$ & -0.153 & 0.219 \\
Smartphone utilization skills & 300 & $2.89 \pm 3.04$ & -1.011 & 0.595 \\
Economic status & 300 & $3.2 \pm 0.75$ & 0.880 & 0.037 \\
Self-rated health status & 300 & $3.18 \pm 0.95$ & -0.307 & 0.248 \\
Number of NCDs & 300 & $2.0 \pm 1.32$ & 0.612 & 0.751 \\
Age (yr) & & & -2.002 & 0.107 \\
65-74 & 158 & 52.7 & & -0.229 \\
$\geq 75$ & 142 & 47.3 & & \\
Sex & & & & \\
Male & 133 & 44.3 & & \\
Female & 167 & 55.7 & & \\
Residence & & & & -1.961 \\
$\quad$ Urban & 159 & 53.0 & & \\
Rural & 141 & 46.0 & & \\
Education level & & & & \\
Uneducated & 47 & 24.7 & & \\
Primary school & 74 & 27.3 & & \\
Secondary school & 82 & 32.3 & & \\
High school or higher & 97 & & & \\
\hline
\end{tabular}

Values are presented as number (\%).

ICT: information and communication technology, NCD: non-communicable disease, SD: standard deviation. 
utilization skills were low, with a mean score of 2.89 out of 10 , but had a relatively high standard deviation of 3.04 .

\section{ICT Use among Older Adults}

Figure 2 presents the distribution of technology usage among participants. More than $65 \%$ of the respondents reported using smartphones. However, fewer than half of the respondents reported using computers or notebooks and the Internet. Wired Internet connections were infrequently used, but more participants used Wi-Fi Internet connections (Figure 2).

Among smartphone users, participants most frequently reported using services such as photo-taking and sharing, followed by social networking services (SNS) and other messaging apps. Searching for information on the Internet and watching videos were common activities for only half of the smartphone users. Participants barely used task management apps, Internet social community activities, and gaming (Figure 3).

\section{Path Analysis of the Factors' Effects on Willingness to} Use Home-Based Healthcare ICT

The results of the path analysis are presented in Table 2 and illuminate the effects of knowledge of new technology and attitudes towards the use of technology, as well as smartphone utilization skills, on older adults' willingness to use home-based healthcare ICT. The results supported H1.2, indicating that knowledge of new technology impacted smartphone utilization skills. H2.1 and H2.3 were also confirmed, as a change of one standardized unit in attitudes towards technology use and smartphone use increased older adults' willingness to use home-based healthcare ICT (Figure 4).

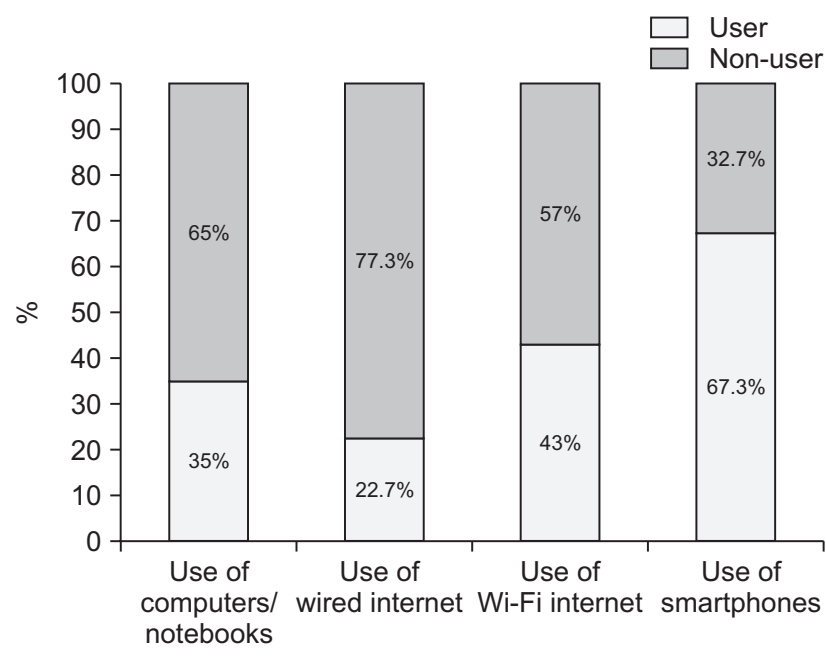

Figure 2. Evaluation of technology use for selected information and communication technology services.
However, knowledge of new technology had no direct impact on the willingness to use home-based healthcare ICT, and attitudes towards technology use had no direct impact on smartphone utilization skills.

No higher changes in coefficients were observed after adjusting the analysis for the control variables. Education, economic status, and self-related health status were found to significantly influence willingness to use home-based healthcare ICT. Participants with more positive attitudes towards using technology, higher smartphone utilization skills, better economic status, and lower self-rated health status tended to be more willing to use ICT.

Table 3 shows the direct, indirect, and total effects of the factors that influenced willingness to use home-based healthcare ICT among older adults. The indirect effect of knowledge of new technology through smartphone utilization was higher than the indirect effect of attitudes towards the use of technology in both models. This indicates that the effect of smartphone use on willingness to use home-based healthcare ICT was more strongly influenced by older adults' knowledge of new technology than by their attitudes towards the use of technology.

\section{Discussion}

The current study was conducted to investigate the relationships of knowledge of new technology and attitudes towards technology use with willingness to use home-based healthcare ICT and the mediating effect of smartphone utilization skills in those relationships. Unlike previous studies in the healthcare field, which more frequently employed the tech-

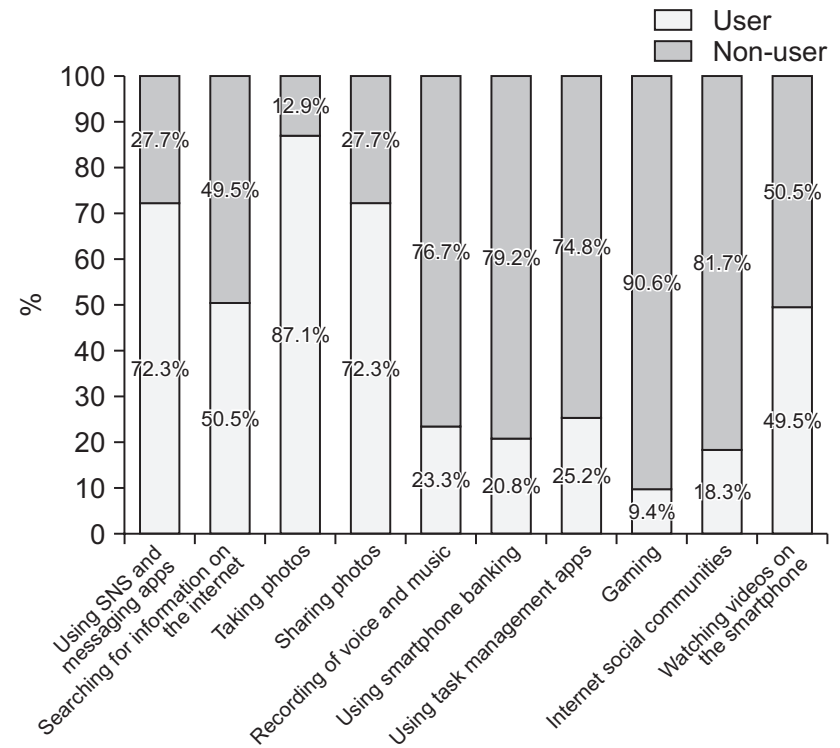

Figure 3. Smartphone utilization skills for various services. 
Table 2. Path analysis of the effects of factors on willingness to use home-based healthcare ICT

\begin{tabular}{|c|c|c|c|c|c|c|}
\hline \multirow{2}{*}{ Path } & \multicolumn{3}{|c|}{ Model 1} & \multicolumn{3}{|c|}{ Model 2} \\
\hline & SRW & CR & $p$-value & SRW & CR & $p$-value \\
\hline $\begin{array}{l}\text { Attitude towards the use of technology } \rightarrow \text { Smartphone } \\
\text { utilization skills }\end{array}$ & 0.047 & 0.911 & 0.357 & 0.047 & 0.911 & 0.362 \\
\hline AI, IoT, and 4IR knowledge $\rightarrow$ Smartphone utilization skills & 0.477 & 9.391 & $<0.001^{\star}$ & 0.477 & 9.391 & $<0.001^{\star}$ \\
\hline $\begin{array}{l}\text { Attitude towards the use of technology } \rightarrow \text { Willingness to } \\
\text { use home-based healthcare ICT }\end{array}$ & 0.188 & 3.504 & $<0.001^{*}$ & 0.172 & 3.313 & $<0.001^{*}$ \\
\hline $\begin{array}{l}\text { AI, IoT, and 4IR knowledge } \rightarrow \text { Willingness to use home- } \\
\text { based healthcare ICT }\end{array}$ & 0.086 & 1.412 & 0.158 & 0.075 & 1.275 & 0.202 \\
\hline $\begin{array}{l}\text { Smartphone utilization skills } \rightarrow \text { Willingness to use home- } \\
\text { based healthcare ICT }\end{array}$ & 0.263 & 4.303 & $<0.001^{\star}$ & 0.246 & 4.163 & $<0.001^{*}$ \\
\hline Sex & - & - & - & 0.099 & 1.912 & 0.056 \\
\hline Age & - & - & - & 0.085 & 1.64 & 0.101 \\
\hline Area of living & - & - & - & -0.001 & -0.024 & 0.981 \\
\hline Education & - & - & - & 0.129 & 2.494 & $0.013^{*}$ \\
\hline Economic status & - & - & - & 0.130 & 2.521 & $0.012^{*}$ \\
\hline Self-rated health status & - & - & - & -0.158 & -3.038 & $0.002^{*}$ \\
\hline Number of NCDs & - & - & - & -0.034 & -0.66 & 0.509 \\
\hline
\end{tabular}

ICT: information and communication technology, AI: artificial intelligence, IoT: Internet of Things, 4IR: Fourth Industrial Revolution, NCD: non-communicable disease, SRW: standardized regression weight, CR: critical ratio. ${ }^{*} p<0.05$.

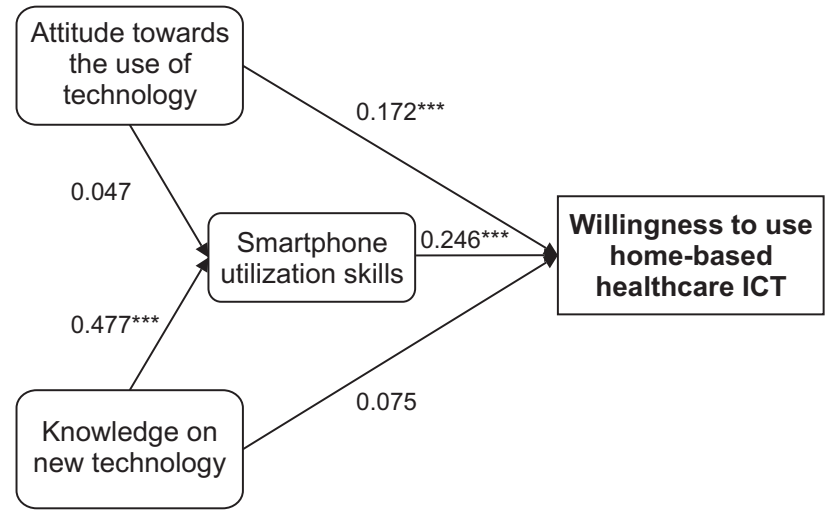

Figure 4. Path diagram of the effects of factors on willingness to use home-based healthcare information and communication technology (ICT).

nology Acceptance model [18] and the unified theory of acceptance and use of technology and its modified models [19], we adapted the IMB model to explore behavior changes based on information, motivation, and use of the services.

The distribution of participants' sociodemographic characteristics was similar to that of previous studies [20]. This study highlighted the influence of attitude towards technology use and smartphone utilization skills on willingness to use home-based healthcare ICT. While knowledge of new technology influenced smartphone utilization skills, that knowledge had only an indirect effect on willingness.

Furthermore, the attitudes of older adults toward technology use were found to stimulate the adoption of new technologies. This finding supports previous studies, according to which a highly positive attitude towards technology use was positively associated with using health applications [21]. Moreover, previous studies have reported that technology anxieties negatively influenced the perception of ICT services' reliability and intention to use smart home technologies among older adults [22]. Along with attitudes, a userfriendly appearance of technologies can contribute to their acceptance [23].

Our finding that smartphone utilization skills had a positive effect on willingness to use home-based healthcare ICT supports previous studies reporting that higher technology use predicted a higher perceived usefulness of technology and higher willingness to use technology [24]. However, Xue et al. [25] did not find a significant relationship between mobile phone usage and intention to use mobile phone-based health interventions among women 50 years or older. This discrepancy in results shows that smartphones and mobile 
Table 3. Total, direct, and indirect effects on willingness to use home-based healthcare ICT among older adults

\begin{tabular}{lccccccc}
\hline \multirow{2}{*}{ Variable } & \multicolumn{3}{c}{ Model 1 } & & \multicolumn{2}{c}{ Model 2 } \\
\cline { 2 - 5 } \cline { 5 - 7 } & Total effect & Direct effect & Indirect effect & Total effect & Direct effect & Indirect effect \\
\hline Attitude towards the use of technology & 0.201 & 0.188 & 0.012 & 0.184 & 0.172 & 0.012 \\
AI, IoT, and 4IR knowledge & 0.212 & 0.087 & 0.125 & 0.193 & 0.075 & 0.117 \\
Smartphone utilization skills & 0.263 & 0.263 & 0 & 0.246 & 0.246 \\
\hline
\end{tabular}

ICT: information and communication technology, AI: artificial intelligence, IoT: Internet of Things, 4IR: Fourth Industrial Revolution.

technologies with more age-friendly functionality may encourage older adults to adopt new technologies [26]. Additionally, the smartphone services predominantly used by older adults were limited to SNS, other messaging services, and photo-taking and sharing, whereas less frequently used services included online information searching and video streaming services. Therefore, education on technology and the usefulness of self-health management smartphone applications could improve older adults' intention to use such technologies.

The socioeconomic characteristics used as control variables showed significant differences among the older population, particularly regarding those with higher education levels. Those with a higher self-rated economic status also reported higher willingness to use home-based healthcare ICT. Furthermore, older adults with a poor self-rated health status were less willing to use healthcare ICT. These findings corroborate other reports on intentions to use new technologies, as well as health ICT and IoT-based technologies in general [20,21].

This study provides an overall understanding of the direct and indirect effects of knowledge of new technology and attitudes towards the use of technology on willingness to use home-based healthcare ICT. The wide introduction of smart home technology and wearable devices for healthcare management has provided more opportunities for older adults to manage their health at home [26]. Most technological solutions for implementing healthcare management systems are based on smartphones [27]. In this sense, developers and providers of home-based healthcare management technologies should consider the attitudes of older adults towards technology use and their level of technology adoption to provide suitable and user-friendly services. Furthermore, socioeconomic factors play an important role in older adults' decisions on using these technologies. For example, previous studies have reported that the cost of the technology and payment methods play an important role in the decision to use ICT for healthcare management [10,22]. Finally, providing education and training on health management technolo- gies could facilitate easy and faster adoption of such technologies and contribute to the improvement of their use [28]. Previous studies on digital and eHealth literacy showed that educational interventions improved older adults' eHealth literacy [29] and that existing users of technologies were more open to adopting and using eHealth [30]. Therefore, additional policy interventions are suggested to promote digital literacy and increase the utilization of ICT for healthcare management among older adults. Furthermore, service customization and education programs should be provided to older adults to increase their utilization of technology and foster positive attitudes towards using ICT.

Despite these implications, this study has several limitations. First, the current study was conducted through the phone, which could have a biasing effect in that participants with low ICT use perceptions might have been omitted. Another limitation of our study is that the survey participants included only residents of Gangwon Province (Republic of Korea); thus, the generalizability of these results to the entire country or other countries may be limited. Further studies should include participants from different regions and countries to improve generalizability.

In conclusion, our findings illuminate how older adults perceive home-based ICT products according to their current experience, knowledge, and services that they need at home for self-health management. This study reveals the necessity and impact of knowledge, attitudes towards use, and actual use of ICT on older adults' willingness to use new technologies for home-based healthcare services. These findings can be helpful in the development of new technologies, educational interventions to boost the adoption of technology among older adults, and future policy developments to respond to the population aging issue.

\section{Conflict of Interest}

No potential conflict of interest relevant to this article was reported. 


\section{Acknowledgments}

This research was supported by the Basic Science Research Program through the National Research Foundation of Korea (NRF), funded by the Ministry of Science and Information Communication Technology (No. NRF2019R1A2C1005840).

\section{ORCID}

Heui Sug Jo (https://orcid.org/0000-0003-0245-3583)

Yu Seong Hwang (https://orcid.org/0000-0001-8934-3533)

Yuliya Dronina (https://orcid.org/0000-0002-5912-3553)

\section{References}

1. Lim T. Population aging in Korea: macroeconomic impacts and financing national health insurance. Korean Econ Rev 2016;32(2):355-82.

2. Abrashkin KA, Zhang J, Poku A. Acute, post-acute, and primary care utilization in a home-based primary care program during COVID-19. Gerontologist 2021;61(1): 78-85.

3. World Health Organization. World report on ageing and health. Geneva, Switzerland: World Health Organization; 2015.

4. Cardozo L, Steinberg J. Telemedicine for recently discharged older patients. Telemed J E Health 2010;16(1): 49-55.

5. Jasemian Y. Elderly comfort and compliance to modern telemedicine system at home. Proceedings of 2008 2nd International Conference on Pervasive Computing Technologies for Healthcare; 2018 Jan 30-Feb 1; Tampere, Finland. p. 60-3.

6. Melkas H, Hennala L, Pekkarinen S, Kyrki V. Impacts of robot implementation on care personnel and clients in elderly-care institutions. Int J Med Inform 2020;134: 104041.

7. Can WS, Seibt SD. Social robotics, elderly care, and human dignity: a recognition-theoretical approach. In: Seibt J, editor. What social robots can and should do. Amsterdam, The Netherlands: IOS Press; 2016. p. 15563.

8. Mshali H, Lemlouma T, Moloney M, Magoni D. A survey on health monitoring systems for health smart homes. Int J Ind Ergon 2018;66:26-56.

9. Pal D, Triyason T, Funikul S. Smart homes and quality of life for the elderly: a systematic review. Proceedings of 2017 IEEE International Symposium on Multimedia (ISM); 2017 Dec 11-13; Taichung, Taiwan. p. 413-9.

10. Liu L, Stroulia E, Nikolaidis I, Miguel-Cruz A, Rincon AR. Smart homes and home health monitoring technologies for older adults: a systematic review. Int J Med Inform 2016;91:44-59.

11. Guner H, Acarturk C. The use and acceptance of ICT by senior citizens: a comparison of technology acceptance model (TAM) for elderly and young adults. Univers Access Inf Soc 2020;19(2):311-30.

12. Kapadia V, Ariani A, Li J, Ray PK. Emerging ICT implementation issues in aged care. Int J Med Inform 2015; 84(11):892-900.

13. Schreurs K, Quan-Haase A, Martin K. Problematizing the digital literacy paradox in the context of older adults' ICT use: aging, media discourse, and self-determination. Can J Commun 2017;42(2):359-377.

14. Agudo-Prado S, Pascual-Sevillana MA, Fombona-Cadavieco J. Uses of digital tools among the elderly. Revista Comunicar 2012;20(39):193-201.

15. Fisher JD, Fisher WA. The information-motivationbehavioral skills model. In: DiClemente RJ, Crosby RA, Kegler MC, editors. Emerging theories in health promotion practice and research: strategies for improving public health. San Francisco (CA): Jossey-Bass; 2002. p. 4070.

16. Heerink M. Exploring the influence of age, gender, education and computer experience on robot acceptance by older adults. Proceedings of 2011 6th ACM/IEEE International Conference on Human-Robot Interaction (HRI); 2011 Mar 8-11; Lausanne, Switzerland. p. 147-8.

17. Wolf EJ, Harrington KM, Clark SL, Miller MW. Sample size requirements for structural equation models: an evaluation of power, bias, and solution propriety. Educ Psychol Meas 2013;76(6):913-34.

18. Rahimi B, Nadri H, Lotfnezhad Afshar H, Timpka T. A systematic review of the technology acceptance model in health informatics. Appl Clin Inform 2018;9(3):60434.

19. Vassli LT, Farshchian BA. Acceptance of health-related ICT among elderly people living in the community: a systematic review of qualitative evidence. Int J Hum Comput Interact 2018;34(2):99-116.

20. Oh YS, Choi EY, Kim YS. Predictors of smartphone uses for health information seeking in the Korean elderly. Soc Work Public Health 2018;33(1):43-54.

21. Rasche P, Wille M, Brohl C, Theis S, Schafer K, Knobe M, et al. Prevalence of health app use among older adults in 
Germany: national survey. JMIR Mhealth Uhealth 2018; 6(1):e26.

22. Pal D, Funilkul S, Charoenkitkarn N, Kanthamanon P. Internet-of-things and smart homes for elderly healthcare: an end user perspective. IEEE Access 2018;6: 10483-96.

23. Mann JA, MacDonald BA, Kuo IH, Li X, Broadbent E. People respond better to robots than computer tablets delivering healthcare instructions. Comput Human Behav 2015;43:112-7.

24. Castilla D, Botella C, Miralles I, Breton-Lopez J, Dragomir-Davis AM, Zaragoza I, et al. Teaching digital literacy skills to the elderly using a social network with linear navigation: a case study in a rural area. Int J Hum Comput Stud 2018;118:24-37.

25. Xue L, Yen CC, Chang L, Chan HC, Tai BC, Tan SB, et al. An exploratory study of ageing women's perception on access to health informatics via a mobile phonebased intervention. Int J Med Inform 2012;81(9):637-48.
26. Kim HS, Lee KH, Kim H, Kim JH. Using mobile phones in healthcare management for the elderly. Maturitas 2014;79(4):381-8.

27. Majumder S, Aghayi E, Noferesti M, Memarzadeh-Tehran H, Mondal T, Pang Z, et al. Smart homes for elderly healthcare-recent advances and research challenges. Sensors (Basel) 2017;17(11):2496.

28. Zhao X, Wang L, Ge C, Zhen X, Chen Z, Wang J, et al. Smartphone application training program improves smartphone usage competency and quality of life among the elderly in an elder university in China: a randomized controlled trial. Int J Med Inform 2020;133:104010.

29. Xie B. Improving older adults' e-health literacy through computer training using NIH online resources. Libr Inf Sci Res 2012;34(1):63-71.

30. Currie M, Philip LJ, Roberts A. Attitudes towards the use and acceptance of eHealth technologies: a case study of older adults living with chronic pain and implications for rural healthcare. BMC Health Serv Res 2015;15:162. 